\title{
A New Genetic Algorithm Based Lane-By-Pass Approach for Smooth Traffic Flow on Road Networks
}

\author{
Shailendra Tahilyani \\ Department of Electronics \& Communication \\ Babu Banarasi Das University \\ Lucknow, India
}

\author{
Manuj Darbari and Praveen Kumar Shukla \\ Department of Computer Science \& Engineering \\ Babu Banarasi Das University \\ Lucknow, India
}

\begin{abstract}
Traffic congestion in urban areas is a very critical problem and increasing day-by-day due to increment in number of vehicles and un-expandable traffic infrastructure. Several intelligent control systems have been developed to deal with this issue. In this paper, a new lane bypass algorithm has been developed for route diversion resulting in smooth traffic flow on the urban road networks. Genetic algorithms are utilized for the parameter optimization in this approach. Finally, the results of the proposed approach are found satisfactory.
\end{abstract}

Keywords- Genetic Algorithms, Fuzzy Logic, Neural Network, Activity Theory.

\section{INTRODUCTION}

Urban Road traffic congestion [1] is a situation that is characterized by high number of vehicles on a road that leads to low speed and longer time taken for a journey. Population growth and exponential growth in number of vehicles have increased the problem of congestion on the country's freeways and highways. Traffic congestion can be classified in two types recurring and non-recurring [2]. Recurring congestion is associated with expected delays, resulting from large number of vehicles at the same time (during peak commuting) at the same place (at busy intersection).Non-recurring congestion is associated with unpredictable delays that are created by spontaneous traffic incidents, such as accidents.

The development of control systems to deal with the congestion for smooth flow of Traffic in urban areas is a critical research issue. Several conventional methods [3-10] have been applied to reduce the problem of traffic congestion. Some of these are, road pricing, supporting the green traffic, parking enforcement, fuel levies, expansion of existing road network, elimination of roundabout and many more. But due to non-linear and unpredictable nature of the traffic movement and the high cost associated with the expansion of existing infrastructure of road networks, the conventional methods are not found very suitable.

Meanwhile, the technology has been integrated to develop some intelligent control systems to deal with the traffic congestion issues, more specifically in urban areas.

Different approaches have been integrated to model and simulate the real time traffic control system, like activity theory [11-13], neural network [14-16], fuzzy logic [17-18], petri nets [19-20], genetic algorithms [21] and their hybrid approaches.

This paper has been divided into 4 sections. In section II, the basic concepts of the proposed system are described. The new proposed algorithm is discussed in Section III. The results analysis of the proposed systems is carried out in the Section IV. Section V is the conclusion and future scope of the proposed approach.

\section{BASIC CONCEPTS FOR PROPOSED SYSTEM}

The Road Network in the urban areas can be considered as a graph. In this graph, edges are classified into two categories: 1. Major Lanes, 2. Minor Sublanes. During peak hours of the traffic movement, the Minor Sublanes can be utilized to overcome any situation of traffic congestion on the major roads of the road network. The prediction of the traffic congestion and transfer of this information is carried out by the proposed algorithm. The information passing and presentation are done by the VMS (Variable Message Sign Board).

A sample road network in the Lucknow City is presented in Fig. 1.

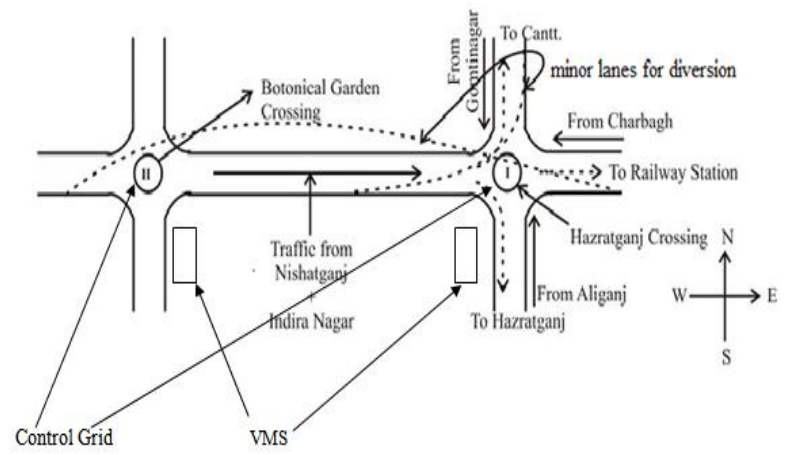

Fig. 1 A Sample Road Network in the Lucknow City

The major activities in the proposed approach are identified as follows:

1) Identification of Route Network in the city, showing the relationship between all the roads (routes). 
2) Identification of Traffic Jams and then identification of alternative minor sub lane route information.

3) Coordination among all the decision (alternative traffic routes) to ensure the smooth traffic flow from source to destination.

The road network is described graph with links $z \in Z$ and junctions $j \in J$. For each signalized junction $\mathrm{j}$, we define the sets of incoming $\mathrm{I}_{\mathrm{j}}$ and outgoing $\mathrm{O}_{\mathrm{j}}$ links. The signal control plan of junction $\mathrm{j}$ is based on a fixed number of stages that belong to the set, whereas $\mathrm{v}_{2}$ denotes the set of stages where link 2 has right of way. Finally the saturation flow $S_{2}$ of link $z \in Z$ and the turning movement rates $\mathrm{t}_{\mathrm{x}, \mathrm{y}}$, where $x \in I_{i}$ and $y \in O_{j}$, are assumed to be known and constant.

By definition, the constraint

$\sum_{i \in F_{n}} g_{n, m}+L_{n}=C$ holds at junction $\mathrm{n}$, where $g_{n, m}$ is the green time of state $m$ at junction $n$ and $L_{n}$ is the total lost time at junction $\mathrm{n}$. It follows the constraints,

$$
g_{m, n} \geq g_{n, m, \min } m \in F_{n}
$$

Consider the following $\mathrm{N}$ Dimensional road network for result analysis in this paper.

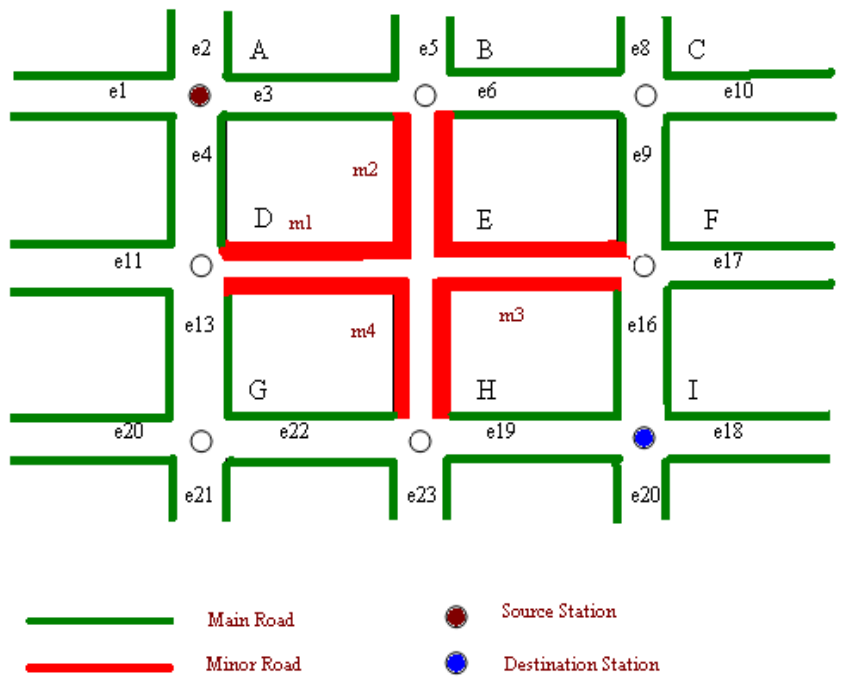

Fig. 2 N Dimensional Road Network

A, B, C.......I are the junctions(Places) at the traffic road network( and connected by road connectives(Transition). The modeling of each junction will be done as discussed in the first section. The phases and movements will be done in the same way.

The proposed approach for route diversion and selection is based on the Genetic Algorithm, an evolutionary techniques for the purpose of optimization in complex ill-defined search spaces. Genetic Algorithms are search and optimization techniques based on Darwinian's principle of Natural Selection. The basic idea behind the natural selection is "select the best, discard the rest". The optimization strategies by genetic algorithms are implemented by simulating evolution of species through the natural selection.

The fundamental technique behind the GA includes the three steps: 1. Evaluation of individual fitness, 2. Formation of gene pool, intermediate population through the selection mechanism, 3. Recombination through the crossover and mutation operators.

The working of the GA can be well expressed by the following data flow diagram, clearly.

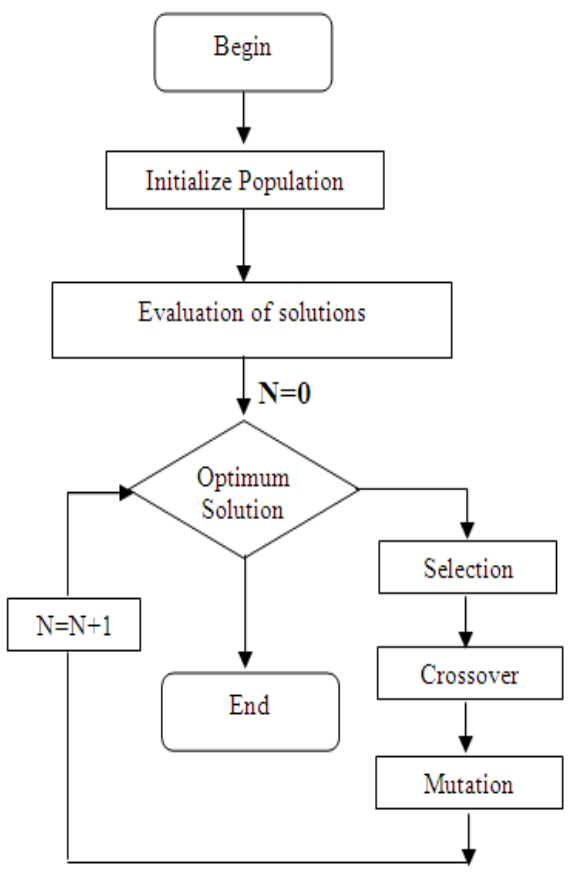

Fig. 3 Working of Genetic Algorithms

\section{PROPOSED SYSTEM}

The problem is identified and mathematically formulated as:

Set of junctions $\quad J=(A, B, C, D, E, F, G, H, I)$

Set of connectives $E=\left(e_{1}, e_{2}, e_{3} \ldots \ldots \ldots e_{23}\right)$

Set of Minor Sublanes M= (m1, m2, m3, m4)

The traffic flow will be decided by the set of connectives from source to destination. Few of the connectives are the bypass connectives that are used by transfer office by pass when the major connectives are full with traffic jam.

In this approach, the VMS technology is used. The commuters will find the signal for route diversion before one crossing through minor sub lane generation if there is any traffic jam at the next crossing. But overall it is also considered that the path from source to destination should be as minimum as possible.

To deal with this problem, the sensors will be placed at each junction for intimating the traffic jam to the previous junction by VMS.

Let the commuter is travelling from station A to I as shown Fig. 4. Now consider the situation, where the commuter 
identifies traffic congestion at junction G through VMS where he is on the route 4 at junction D. Now to deal with this situation the control system would generate the signal for route diversion through minor lane by pass and the commuter would get the signal to choose the route $\mathrm{m} 1$ and $\mathrm{m} 4$ to reach his destination.

The procedure of route diversion should be integrated with the approach traffic phase and movement decision approaches.

\section{A. Algorithm ROUTE DIVERSION through minor sub-lane}

For the route diversion the proposed algorithm is as follows:

ROUTE DIVERSION $\left(\alpha_{V M S}, \alpha M_{V M S}\right)$

1. Start

2. Generate $\alpha_{\mathrm{VMS}}$ for each junction at different intervals of time.

$\alpha_{\mathrm{VMS}}=$ Traffic Flow $/$ Mean Velocity

The value of Traffic Flow will be estimated by the

Sensors and Mean Velocity is calculated at different time intervals.

Repeat steps 3 to 5 until the desired destination,

3. Collect the values of $\alpha_{\mathrm{VMS}}$ (update them on time intervals) for all junctions in different paths from a specified source to destination, at a control centre.

4. Generate the maximum value of $\alpha_{\mathrm{VMS}}$ from which the traffic congestion will start and it is denoted by $\alpha \mathrm{M}_{\mathrm{VMS}}$.

5. Apply minimization function $F_{\min }$ on the values of $\alpha_{\mathrm{VMS}}$ to get the next node, iteratively.

6. If $F_{\text {min }}\left(\alpha_{\mathrm{VMS}}, \alpha \mathrm{M}_{\mathrm{VMS}}\right) \geq \alpha_{\mathrm{VMS}}$ then

Send the signal to previous junction's VMS for route diversion, if the selected junction is different from the regular path,

otherwise

Follow the main route decided previously.

7. End.

\section{B. GA ROUTE Diversion Algorithm}

This route diversion process can be seen as an optimization problem and its extended version can be produced using genetic algorithm. This proposal is as follows:

\section{GA-ROUTE DIVERSION $\left(\alpha_{V M S}, \alpha M_{V M S}\right)$}

\{

Initialize all the paths as first population

$$
\left(\mathrm{e}_{1}, \mathrm{e}_{2}, \ldots \ldots \ldots \ldots \ldots, \mathrm{e}_{\mathrm{n}}\right)
$$

Evaluate the fitness of the each path as per the function

$$
\mathrm{F}_{\text {min }}\left(\alpha_{\mathrm{VMS}}, \alpha \mathrm{M}_{\mathrm{VMS}}\right)
$$

Set the termination criteria

$$
\mathrm{F}_{\text {min }}\left(\alpha_{\mathrm{VMS}}, \alpha \mathrm{M}_{\mathrm{VMS}}\right) \geq \alpha_{\mathrm{VMS}}
$$

while the termination criteria is satisfied$$
\{
$$

Select the most optimized path

Crossover (Various combinations of paths)
Mutation (Change a particular path in the route)

Evaluate the new population

\}

\section{RESUlt ANALYSIS}

The two cases are considered here for result analysis. Let the source station is A and destination station is I.

The selected route by commuter is (A, B, C, F, I). Now according to the space capacity of the junction, $\alpha \mathrm{M}_{\mathrm{VMS}}=9.5$ (for junction $\mathrm{C}$ ). Here, TABLE I shows the traffic flow, mean velocity and calculated values of $\boldsymbol{\alpha}_{\mathrm{vms}}$. at different time intervals in a day.

TABLE I : TRAFFIC FLOW \& MEAN VELOCITY [JUNCTION C]

\begin{tabular}{|c|c|c|c|}
\hline Time & Traffic Flow & Mean velocity $(\mathbf{k m} / \mathbf{h})$ & $\boldsymbol{\alpha}_{\text {vMs }}$ \\
\hline 7:00 AM & 10 & 60 & 0.16 \\
\hline 7:30 AM & 20 & 40 & 0.50 \\
\hline 8:00 AM & 40 & 30 & 1.33 \\
\hline 8:30 AM & 60 & 25 & 2.40 \\
\hline 9:00 AM & 100 & 20 & 5.00 \\
\hline 9:30 AM & 150 & 15 & 10.00 \\
\hline 10:00AM & 200 & 10 & 20.00 \\
\hline 10:30 AM & 250 & 05 & 50.00 \\
\hline
\end{tabular}

\section{Case 1}

At time 9:00 AM,

$\mathrm{F}_{\min }\left(\alpha_{\mathrm{VMS}}, \alpha \mathrm{M}_{\mathrm{VMS}}\right)=\mathrm{F}_{\min }(5,9.5)<\alpha_{\mathrm{VMS}}$

Hence according to the algorithm, no diversion is required through the minor sub lane by- pass. This case is shown in Figure 4.

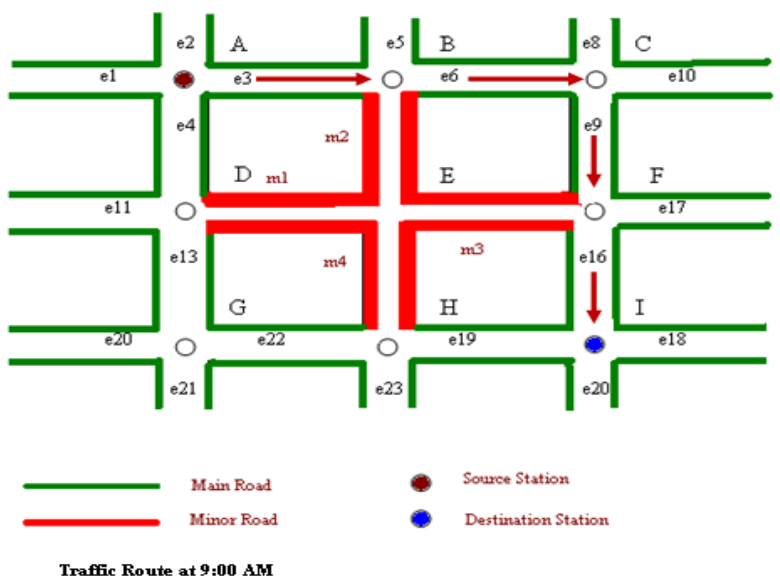

Fig. 4 Case 1

Case 2 At time 9:30 AM,

$\mathrm{F}_{\text {min }}\left(\alpha_{\mathrm{VMS}}, \alpha \mathrm{M}_{\mathrm{VMS}}\right)=\mathrm{F}_{\min }(10,9.5)>\alpha_{\mathrm{VMS}}$

Hence according to the algorithm, the minor lane by pass would be generated and the commuter will get the signal to divert the route at minor lane $\mathrm{m} 2$. This case is shown in Figure 5. 

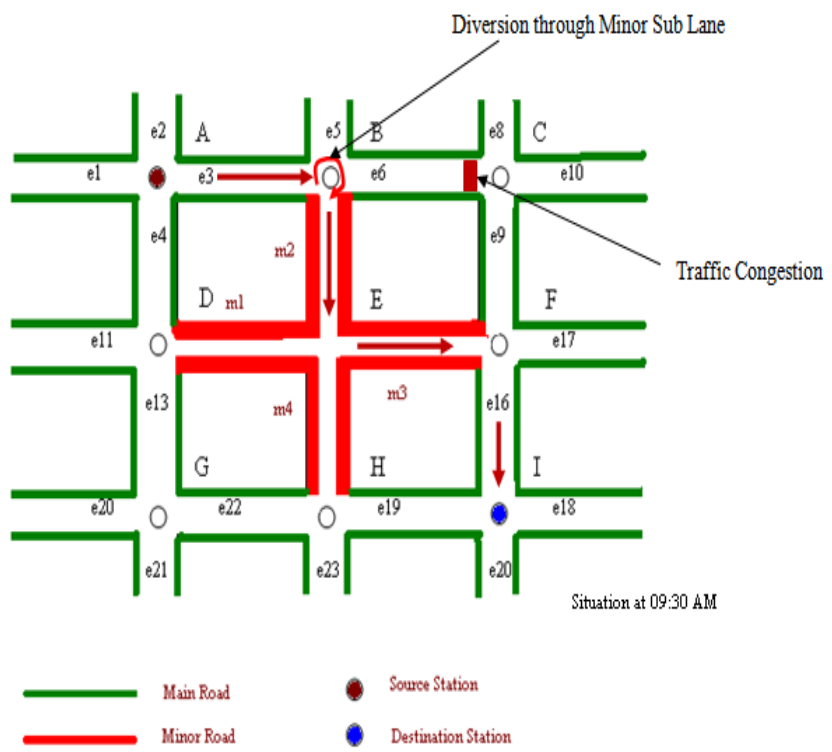

Fig. 5 Case 2

The variations of $\alpha_{\mathrm{VMS}}$ are represented as follows at both the junctions,

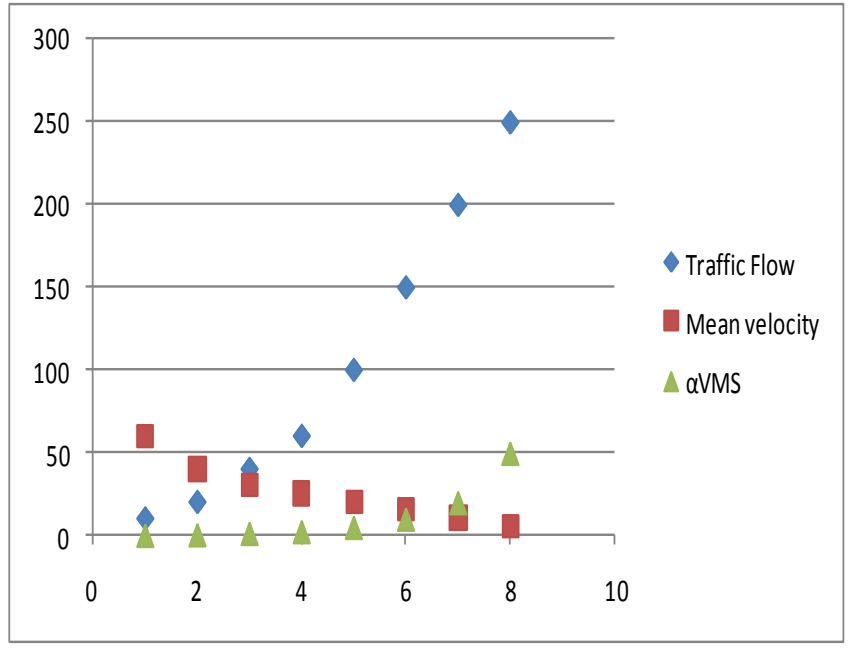

Fig. 6 Traffic Flow, Mean Velocity and $\alpha_{\mathrm{VMS}}$ at Junction C

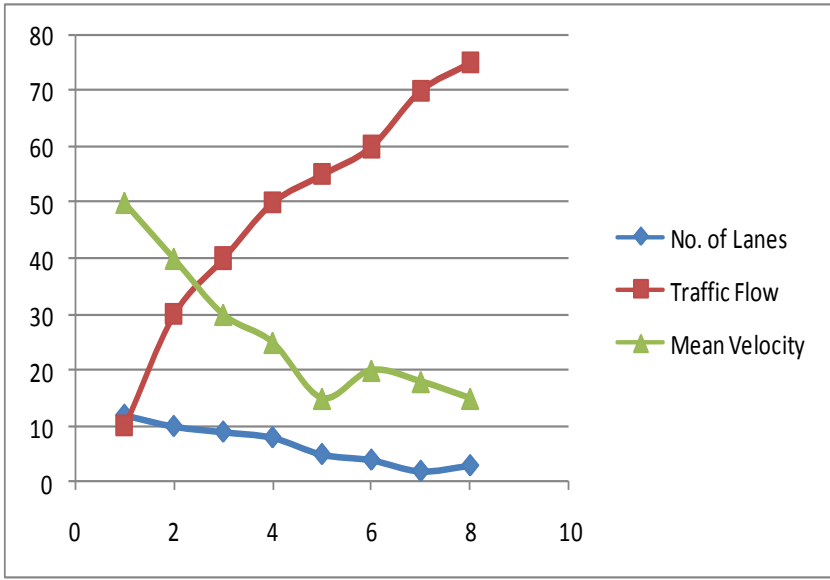

Fig. 7 Simulation result (Showing importance of Minor Role)
The results are shown in Figure 6 and 7 and indicate that the closure of minor roads could improve the travel time. The estimation of routing is usually performed by calculating the feedback costs associated with each link network-wide for every pre-specified interval ( $5 \mathrm{~min}$ in this case). This causes the vehicles to opt for major links, thereby achieving better optimization and lesser travel delay time. However, when multiple lanes were closed, a slight degradation is seen during the first peak traffic period, which subsequently settles down in the later peak periods to a slightly higher delay value than in the normal operation.

\section{CONCLUSION AND Future ScOPE}

This paper introduces a new approach to deal with the traffic congestion on the road networks. A new lane by pass based approach is introduced using genetic algorithms. The results are found satisfactory.

In future, the authors would like to expand this approach for IVHS (Intelligent Vehicle Highway System) by integrating Multi Agent Systems.

\section{REFERENCES}

[1] S. K. Singh, Review of Urban Transportation in India, Journal of Public Transport, vol. 8, no. 1, pp. 79-97, 2005.

[2] MGH Bell, Transport Planning and Traffic Engineering, C. A. O'Flaherty Eds. Butterworth-Heinemamm, Elsevier, 2006

[3] R. Arnott, T. Rave and R. Schöb, Alleviating Urban Traffic Congestion, MIT Press, Sep. 2005.

[4] M. Papageorgiou, C. Diakaki, V. Dinopoulou, A. Kotsialos, Yibing Wang, Review of road traffic control strategies, Proceedings of the IEEE, vol. 91, no. 12, 2043 -2067, 2003.

[5] S.-W. Chiou, Optimization of a non-linear area traffic control system with elastic demand, Automatica, vol. 46, no. 10, pp. 1626-1635, 2010.

[6] D. W. Hearn, M. V. Ramana, Solving congestion toll pricing models equilibrium and advanced transportation modelling, Kluwer Academic, 1998, pp. 109-124.

[7] L. Bai, D. W. Hearn, S. Lawphongpanich, Relaxed toll sets for congestion pricing problems, Mathematical \& Computational models for congestion charging, Springer, 2006.

[8] D W Hearn, M B Yildirim, M V Ramana, L H Bai, Computational methods for congestion toll pricing models, 2001 IEEE Intelligent Transportation Systems Proceedings, pp. 257-262, 2001.

[9] P. T. Blyth, Combatting congestion: the role of road traffic informatics, Traffic Congestion - Engineering the Solution, IEE Colloquium on, 1991, London, UK, pp. 3/1-3/14.

[10] E. Ericsson, H. Carsson, K. Brundellfreij, Optimization route choice for lowest fuel consumption - potential effects of a new driver support tool, Transportation Research Part C: Emerging Technologies, vol. 14, no. 6, pp. 369-383, 2006.

[11] Y. Engestrot, Leaniing, Working and Imagining - Twelve Studies in Activity Theory, Orientakonsultit Oy, Helsinki, 1990.

[12] Y. K. Chin, N. Bolong, S. S. Yang, K. T. K. Teo, Exploring Q-learning optimization in traffic signal timing plan management, Third International Conference on Computational Intelligence, Communication Systems and Networks, 2011, pp. 269-274.

[13] D.-Y. Lin, N. Eluru, S. T. Waller, C. R. Bhat, Evacuation Planning Using the Integrated System of Activity-Based Modeling and Dynamic Traffic Assignment, Transportation Research Record: Journal of the Transportation Research Board, vol. 2132, 2009, pp. 69-77.

[14] S. Carrillo, J. Harkin, L. McDaid, S. Pande, S. Cawley and F. Morgan, Adaptive Routing Strategies for Large Scale Spiking Neural Network Hardware Implementations, Artificial Neural Networks and Machine Learning - ICANN 2011, Lecture Notes in Computer Science, 2011, vol. 6791/2011. 
[15] A. Nagare, S. Bhatia,Traffic Flow Control using Neural Network, International Journal of Applied Information Systems (IJAIS), vol. 1, no. 2, January 2012 .

[16] S. Carrillo, J. Harkin, L. McDaid, S. Pande, S. Cawley, B. McGinley, F. Morgan, Advancing interconnect density for spiking neural network hardware implementations using traffic-aware adaptive network-on-chip routers, Neural Networks, vol. 33, Sept. 2012, pp. 42-57.

[17] S. Mehan, V. Sharma, Development of traffic light control system based on Fuzzy Logic, ACAI '11 Proceedings of the International Conference on Advances in Computing and Artificial Intelligence, 2011.

[18] V. P. Vijayan, B. Paul, Multi Objective Traffic Prediction Using Type-2 Fuzzy Logic and Ambient Intelligence, Advances in Computer
Engineering (ACE), 2010 International Conference on, pp. 309-311, 2010.

[19] G. F. List, M. Setin, Modeling traffic signal control using petri nets, IEEE Transactions on Intelligent Transportation Systems, vol. 5, no. 3, Sept. 2004.

[20] H. Wang, Modeling and analysis of traffic signal systems using Petri nets, Ph.D. dissertation, Dept. Civil Eng., Rensselaer Polytechnic Inst., Troy, NY, 1992.

[21] J. J. Sanchez-Medina, Traffic Signal Optimization in "La Almozara" District in Saragossa Under Congestion Conditions, Using Genetic Algorithms, Traffic Microsimulation, and Cluster Computing, IEEE Transactions on Intelligent Transportation Systems, vol. 11, no. 1, pp. $132-141,2010$. 